\title{
Response of Different Genotypes of Faba Bean Plant to Drought Stress
}

\author{
Manzer H. Siddiqui ${ }^{1, *}$, Mutahhar Y. Al-Khaishany ${ }^{1}$, Mohammed A. Al-Qutami ${ }^{\text {, }}$ \\ Mohamed H. Al-Whaibi ${ }^{1}$, Anil Grover ${ }^{2}$, Hayssam M. Ali ${ }^{1}$, Mona S. Al-Wahibi ${ }^{1}$ and \\ Najat A. Bukhari ${ }^{1}$
}

1 Department of Botany and Microbiology, College of Science, King Saud University, Riyadh 2455, Saudi Arabia; E-Mails: muthr20@gmail.com (M.Y.A.-K.); maqutami@gmail.com (M.A.A.-O.); mwhaibi@ksu.edu.sa (M.H.A.-W.); hayhassan@ksu.edu.sa (H.M.A.); malwhibi@ksu.edu.sa (M.S.A.); najatab@ksu.edu.sa (N.A.B.)

2 Department of Plant Molecular Biology, University of Delhi South Campus, Benito Juarez Road, Dhaula Kuan, New Delhi 110021, India; E-Mail: anil.anilgrover@gmail.com

* Author to whom correspondence should be addressed; E-Mail: manzerhs@yahoo.co.in; Tel.: +966-1-14675-872. Fax: +966-1-14675-833.

Academic Editor: Marcello Iriti

Received: 26 February 2015 / Accepted: 14 April 2015 / Published: 5 May 2015

\begin{abstract}
Drought stress is one of the major abiotic stresses that are a threat to crop production worldwide. Drought stress impairs the plants growth and yield. Therefore, the aim of the present experiment was to select the tolerant genotype/s on the basis of moprpho-physiological and biochemical characteristics of 10 Vicia faba genotypes (Zafar 1, Zafar 2, Shebam, Makamora, Espan, Giza Blanka, Giza 3, C4, C5 and G853) under drought stress. We studied the effect of different levels of drought stress i.e., (i) normal irrigation (ii) mild stress (iii) moderate stress, and (iv) severe stress on plant height (PH) plant $^{-1}$, fresh weight (FW) and dry weight (DW) plant $^{-1}$, area leaf ${ }^{-1}$, leaf relative water content (RWC), proline (Pro) content, total chlorophyll (Total $\mathrm{Chl}$ ) content, electrolyte leakage (EL), malondialdehyde (MDA), hydrogen peroxide $\left(\mathrm{H}_{2} \mathrm{O}_{2}\right)$ content, and activities of catalase (CAT), peroxidase (POD) and superoxide dismutase (SOD) of genotypes of faba bean. Drought stress reduced all growth parameters and Total Chl content of all genotypes. However, the deteriorating effect of drought stress on the growth performance of genotypes "C5" and "Zafar 1" were relatively low due to its better antioxidant enzymes activities (CAT, POD and SOD), and accumulation of Pro and Total Chl, and leaf RWC. In the study,
\end{abstract}


genotype "C5" and "Zafar 1" were found to be relatively tolerant to drought stress and genotypes "G853" and "C4" were sensitive to drought stress.

Keywords: growth parameters; proline; drought stress; Vicia faba; antioxidant enzymes; oxidative stress

\section{Introduction}

Water stress is considered a detrimental factor for the production of crops worldwide. Water scarcity is accentuated by other abiotic stresses as well as global warming [1]. Globally, more that $50 \%$ of the average yield of most major crops is lost due to drought stress (DS) [2]. Today, it has become a challenging task to combat drought stress worldwide. Like other environmental stresses, DS causes a series of physiological, biochemical and molecular changes in plants. Due to water stress, whole plant metabolism is dramatically affected by the over-production of reactive oxygen species (ROS) that are responsible for oxidation of multicellular components like proteins, lipids, DNA and RNA, resulting in death of cells [3]. Water deficit in plants causes inhibition of photosynthesis by altering pathway regulation by stomatal closure and decreasing flow of $\mathrm{CO}_{2}$ into mesophyll tissues and also by impairing the activity of ribulose-1,5-bisphosphate carboxylase/oxygenase [4-6]. Also, respiration, translocation, ion uptake, carbohydrates, nutrient assimilation and growth promoters are disturbed under stress $[7,8]$. Under stress, plants develop a defensive mechanism and cellular homeostasis by the accumulation of osmolytes (i.e., proline, glycinebetaine) and proteins thereby increasing tolerance of plants to stress $[9,10]$. However, plant tolerance to abiotic stresses is a complex trait, involving a range of molecular, biochemical and physiological mechanisms [11]. The response of plants to stresses depends on species and genotypes, the length and severity of water deficit, and age and development stage [12].

Vicia faba, also known as faba bean, broad bean and fava bean, has a long history of cultivation. It is an important winter legume crop that is rich in protein and energy, and used in feed and food. According to FAOSTAT [13], China, Ethiopia, France, Egypt and Australia are main faba bean producing countries. It has significant value in improving the fertility of soil by its rotation cultivation with cereal crops; thereby, fixing nitrogen in symbiosis makes them excellent colonizers of low-N environments. The production of faba bean is not enough to feed the ever-growing world population. There are many biotic and abiotic factors that cause reduced yields. Also, bean plants showed a great magnitude of intraspecific variation [14,15], and molecular and physiological changes occur $[7,8,16]$ for stress tolerance. Faba bean cultivation particularly in arid and semi-arid regions is unsuitable because this crop is not sufficiently drought and heat tolerant as it is susceptible to moisture and high temperature stresses [17]. Keeping in view the importance of this crop for humans as well as animals, the present experiment was planned to study the effect of DS on different genotypes of faba bean plants. The main objective of this experiment was to determine DS tolerant and sensitive genotypes on the basis of physio-morphological and biochemical parameters. 


\section{Results and Discussion}

In this study, the performance of different genotypes was evaluated in terms of plant height (PH) plant ${ }^{-1}$, shoot fresh (SF) and shoot dry (SD) weight plant $^{-1}$, area leaf ${ }^{-1}$, relative water content (RWC), total chlorophyll (Total $C h l$ ), proline (Pro) content, electrolyte leakage (EL), and content of malondialdehyde (MDA) and $\mathrm{H}_{2} \mathrm{O}_{2}$, and activity of catalase (CAT), peroxidase (POD) and superoxide dismutase (SOD). The effect of DS treatments on these parameters of genotypes was found to be significant (Tables 1-4).

The data reveal that growth performance of faba bean genotypes were affected significantly, depending on the level of water deficit (Table 1). In general, drought stress affected all growth parameters (PH, SF weight and SD weight plant, leaf area) of plants of all genotypes. Genotype "C5" exhibited the highest values for $\mathrm{PH}$ and leaf area as compared to the other genotypes under severe drought stress. At severe drought stress, genotype "G853" showed the lowest value for all growth parameters. However, genotype "C5" proved to be the best by giving highest values for all growth characteristics among nine genotypes, and genotype "G853", being at par with genotype "C4" for leaf area, had the lowest values for these parameters. A decrease in growth parameters may be due to the impairment of cell division, cell enlargement caused by loss of turgor, and inhibition of various growth metabolisms $[18,19]$. These results strengthen the findings of Ouzounidou et al. [20] on faba bean, Ali et al. [21] on faba bean; Farooq et al. [8] on rice and Asrar and Elhindi [22] on marigold, who reported that DS reduced plant growth characteristics. Among the cultivars, "C5" was found to be more tolerant by giving highest values for all growth characteristics in comparison to nine genotypes, and genotype "G853" was found to be sensitive to drought stress.

Under drought stress, leaf RWC plays an important role in tolerance of plants to stress by inducing osmotic adjustment due to the accumulation of osmoprotectants [12,23,24]. The maintenance of a high plant water status during stress is an important defensive mechanism to retain enough water by minimizing water loss (e.g., caused by stomatal closure, trichomes, reduced leaf area, senescence of older leaves, etc.) and maximizing water uptake (e.g., by increased root growth) [12]. In the present experiment, Tables 2 and 3 depict that leaf RWC, Pro accumulation, Total $C h l$ content, electrolyte leakage, and content of MDA and $\mathrm{H}_{2} \mathrm{O}_{2}$ of all genotypes were significantly affected by water stress. At increasing levels of drought stress, leaf RWC and Total Chl content decreased inversely. The differences in RWC in all genotypes could be associated with their ability of water absorption from soil. Thus, we conclude that genotype "C5" could have better ability to resist drought stress. According to Khanna-Chopra \& Selote [25], under stress, the drought-resistant wheat plants exhibited better leaf water relations in terms of turgor potential and RWC as compared to sensitive genotypes. Genotype "C5", being at par with genotype "Giza 3", had the highest RWC under severe water stress conditions.

Pro accumulation increased with increasing levels of water stress (Table 2). The accumulation of Pro in plants reduces the toxic effects of ions on enzymes activity and also lowers the generation of free radicals formed by drought stress. Also, Pro associated with recovery resistance by serving a source of respiratory energy to the plants under stress [26]. Under severe DS, genotype "C5" gave the maximum value for Pro content, and genotype "G853", being at par with genotype "Giza 3", exhibited lower value for content of Pro. Also, genotype "C5", followed by genotypes "Zafar 1" and "Zafar 2", had the maximum value for Pro content (Table 2). 
Under severe drought stress, genotypes "Zafar 1", followed by genotypes "C5", "Giza 3" and "Makamora", gave the maximum value for Total Chl content (Table 2). This result strongly supports the findings of Ali et al. [21] in faba bean, and Mafakheri et al. [27] in chickpea genotypes. A decrease in Total Chl content may be due to the activity of chlorophyllase, a chlorophyll degrading enzyme [28]. Under drought stress, a low inhibition of Total Chl synthesis in genotypes Zafar 1', "C5" and "Giza 3" could be associated with better light harvesting efficiency, thereby improving dry matter production (Table 1).

We observed that electrolyte leakage, and accumulation of MDA and $\mathrm{H}_{2} \mathrm{O}_{2}$ were found to be dependent on the severity of drought stress (Table 3). Genotype "C5", being at par with genotype "Zafar 1" for electrolyte leakage, genotypes "Zafar 1", "Giza Blanka" and Zafar 2 for MDA accumulation, gave the lowest values under severe condition of drought stress. Also, genotype "C5" showed the lowest content of $\mathrm{H}_{2} \mathrm{O}_{2}$ under water stress condition. All three oxidative stress indicators (electrolyte leakage, and accumulation of MDA and $\mathrm{H}_{2} \mathrm{O}_{2}$ ) were found to be almost lower in genotype "C5" and the highest in cultivars "G853", "C4" and "Makmora". These results agree with the findings of Ouzounidou et al. (20), Terzi and Kadioglu [29]; Ali et al. [21] and Quan et al. [30]. According to Jiang and Huang [31], accumulation of MDA affects the RWC and photosynthetic pigment of plants. Among the cultivars, "C5" had the lowest values for theses parameters. These results reveal that tolerance of genotype "C5" to drought stress could be positively related to leaf RWC and synthesis of Total Chl (Table 2).

In general, activity of antioxidant enzymes (CAT, POD, and SOD) were significantly increased with increasing levels of drought stress in plants of all genotypes, (Table 4). Under severe water stress conditions, genotype "Shebam 1", being at par with genotype "C5", gave a higher value for CAT activity. However, the highest enzymes activities were noted in genotypes "C5" for POD and SOD at severe level of water stress. Genotype "Zafar 1" followed by genotype "C5" for the activity of POD and SOD. Moreover, the magnitude of increase in these enzymes' activity in genotypes "C5" was higher than other genotypes of faba bean under DS, except genotypes "Shebam 1" for CAT. Genotype "G853", followed by genotype "C4", exhibited the lowest enzyme activity under water stress. As we know, abiotic stress leads to the generation of reactive oxygen species (ROS: Superoxide anion radicals, hydroxyl radicals, $\mathrm{H}_{2} \mathrm{O}_{2}$, alkoxy radicals and singlet oxygen) that may react with a large variety of biomolecules - such as deoxyribonucleic acid, protein, lipids and carbohydrates - Causing lipid peroxidation linked membrane deterioration $[32,33]$. To overcome oxidative damage, plants develop an antioxidant system to scavenge ROS. In the present experiment, activity of antioxidant enzymes (POD, CAT and SOD) in plants of all genotypes increased under drought stress (Table 4). However, under DS, the highest enzymes activities were noted in genotypes "C5", "Zafar 1", and genotypes "G853" and "C4" exhibited the lowest value. Thus, it could be possible and reasonable to suggest that genotypes "C5" and "Zafar 1" were more tolerant than the other genotypes, because the maximum values for these enzymes' activity were recorded (Table 4). 
Table 1. Growth performance of faba bean genotypes under drought stress.

\begin{tabular}{|c|c|c|c|c|c|c|c|c|c|c|}
\hline \multirow{2}{*}{ Treatments } & \multicolumn{10}{|c|}{ Genotypes } \\
\hline & Zafar 1 & Zafar 2 & Giza Blanka & Espan & Makmora & Shebam 1 & Giza 3 & $\mathrm{C} 4$ & $\mathbf{C 5}$ & G853 \\
\hline \multicolumn{11}{|c|}{ Plant Height $(\mathrm{cm})$ Plant $^{-1}$} \\
\hline Control & $56.00 \pm 0.58^{\mathrm{ijk}}$ & $59.00 \pm 0.58$ ghi & $61.00 \pm 0.58^{\mathrm{fgh}}$ & $54.33 \pm 1.20^{\mathrm{jkl}}$ & $47.33 \pm 1.20^{\text {no }}$ & $64.33 \pm 1.86^{\text {ef }}$ & $58.00 \pm 1.53^{\mathrm{hij}}$ & $79.00 \pm 0.58^{\mathrm{c}}$ & $107.67 \pm 1.45^{\mathrm{a}}$ & $36.67 \pm 0.88^{\mathrm{st}}$ \\
\hline Mild & $45.67 \pm 1.76^{\mathrm{op}}$ & $56.00 \pm 0.58^{\mathrm{ijk}}$ & $57.33 \pm 1.45^{\text {hij }}$ & $52.00 \pm 1.15^{\mathrm{lm}}$ & $31.33 \pm 0.33$ uv & $62.00 \pm 1.53^{\mathrm{fg}}$ & $40.00 \pm 1.73$ qrs & $70.33 \pm 0.33^{\mathrm{d}}$ & $86.67 \pm 0.88^{b}$ & $25.67 \pm 0.88^{\mathrm{w}}$ \\
\hline Moderate & $37.33 \pm 1.20^{\mathrm{rs}}$ & $42.33 \pm 1.45^{\mathrm{pq}}$ & $50.00 \pm 1.00^{\mathrm{mn}}$ & $45.33 \pm 1.76^{\mathrm{op}}$ & $30.00 \pm 1.53$ uv & $39.00 \pm 1.73$ qrs & $52.67 \pm 1.86^{\mathrm{klm}}$ & $66.67 \pm 0.88^{\mathrm{e}}$ & $73.67 \pm 0.33^{d}$ & $19.00 \pm 0.58^{\mathrm{x}}$ \\
\hline Severe & $28.67 \pm 1.67^{\mathrm{vw}}$ & $38.67 \pm 0.33^{\mathrm{qrs}}$ & $33.67 \pm 0.88^{\text {tu }}$ & $41.00 \pm 1.73^{\mathrm{qr}}$ & $21.67 \pm 0.88^{\mathrm{x}}$ & $28.33 \pm 1.20^{\mathrm{vw}}$ & $32.67 \pm 1.76^{\mathrm{u}}$ & $58.00 \pm 0.58^{\text {hij }}$ & $66.67 \pm 0.88^{\mathrm{e}}$ & $13.67 \pm 0.33^{y}$ \\
\hline \multicolumn{11}{|c|}{ Shoot FW (g) Plant ${ }^{-1}$} \\
\hline Control & $5.63 \pm 0.48^{\mathrm{cd}}$ & $5.10 \pm 0.12^{\text {cdef }}$ & $4.87 \pm 0.44^{\text {cdefgh }}$ & $5.70 \pm 0.10^{\mathrm{cd}}$ & $5.13 \pm 0.03^{\text {cdef }}$ & $5.47 \pm 0.78^{\mathrm{cd}}$ & $5.00 \pm 0.06^{\text {cdefg }}$ & $6.00 \pm 0.31^{\mathrm{c}}$ & $8.50 \pm 0.23^{\mathrm{a}}$ & $2.40 \pm 0.06^{\text {nopqrs }}$ \\
\hline Mild & $2.97 \pm 0.87^{\mathrm{klmnopqr}}$ & $3.53 \pm 0.82^{\text {hijklmno }}$ & $4.70 \pm 0.32^{\text {defghij }}$ & $4.53 \pm 0.35^{\text {defghi }}$ & $4.73 \pm 0.12^{\text {cdefgh }}$ & $3.77 \pm 0.43^{\text {fghijklm }}$ & $4.07 \pm 0.46^{\text {efghijk }}$ & $4.87 \pm 0.12^{\text {cdefgh }}$ & $7.17 \pm 0.15^{b}$ & $2.20 \pm 0.06^{\text {opqrst }}$ \\
\hline Moderate & $2.13 \pm 0.52^{\text {pqrst }}$ & $2.03 \pm 0.29^{\mathrm{pqrst}}$ & $4.07 \pm 0.50^{\text {efghijk4 }}$ & $3.13 \pm 0.83^{\text {jklmnopq }}$ & $1.80 \pm 0.38$ qrst & $3.70 \pm 0.15^{\text {ghijklmn }}$ & $3.90 \pm 0.21^{\text {efghijkl }}$ & $4.00 \pm 0.15^{\text {efghijkl }}$ & $5.27 \pm 0.09^{\text {cde }}$ & $1.43 \pm 0.24^{\text {stu }}$ \\
\hline Severe & $1.67 \pm 0.35^{\text {rstu }}$ & $2.43 \pm 0.75^{\text {mnopqrs }}$ & $3.27 \pm 0.32^{\mathrm{ijk} k m n o q}$ & $2.67 \pm 0.60^{\text {Imnopqrs }}$ & $0.90 \pm 0.25^{\text {tu }}$ & $1.97 \pm 0.49^{\text {pqrst }}$ & $3.17 \pm 0.43^{\mathrm{ijk} k m n o p}$ & $2.70 \pm 0.06^{\text {Imnopqrs }}$ & $4.37 \pm 0.15^{\text {defghij }}$ & $0.50 \pm 0.06^{\mathrm{u}}$ \\
\hline \multicolumn{11}{|c|}{ Shoot DW (g) Plant ${ }^{-1}$} \\
\hline Control & $0.76 \pm 0.16^{\mathrm{ab}}$ & $0.63 \pm 0.06^{\text {abcdef }}$ & $0.70 \pm 0.23 \mathrm{abc}$ & $0.73 \pm 0.07^{\mathrm{ab}}$ & $0.59 \pm 0.02^{\text {bcdefg }}$ & $0.56 \pm 0.07^{\text {bcdefgh }}$ & $0.57 \pm 0.02^{\text {bcdefgh }}$ & $0.56 \pm 0.04^{\text {bcdefgh }}$ & $0.83 \pm 0.03^{\mathrm{a}}$ & $0.40 \pm 0.01^{\text {ghjjkln }}$ \\
\hline Mild & $0.46 \pm 0.02$ efghijk & $0.40 \pm 0.12^{\text {ghijklm }}$ & $0.67 \pm 0.06$ abcde & $0.50 \pm 0.01^{\text {cdefghi }}$ & $0.46 \pm 0.07^{\text {efghijkl }}$ & $0.44 \pm 0.06^{\text {fghijklm }}$ & $0.45 \pm 0.06^{\text {fghijklm }}$ & $0.37 \pm 0.07^{\text {ghijklm }}$ & $0.68 \pm 0.02$ abcd & $0.29 \pm 0.01^{\mathrm{ijklm}}$ \\
\hline Moderate & $0.41 \pm 0.01^{\text {fghijklm }}$ & $0.30 \pm 0.09^{\mathrm{ij} \mathrm{jkm}}$ & $0.46 \pm 0.05^{\text {efghijkl }}$ & $0.45 \pm 0.05^{\text {fghijklm }}$ & $0.36 \pm 0.02^{\text {hijklm }}$ & $0.42 \pm 0.06^{\text {fghijklm }}$ & $0.47 \pm 0.03^{\text {defghij }}$ & $0.25 \pm 0.00^{\mathrm{klm}}$ & $0.50 \pm 0.01$ cdefghi & $0.24 \pm 0.07^{\mathrm{lm}}$ \\
\hline Severe & $0.30 \pm 0.01^{\mathrm{ijklm}}$ & $0.23 \pm 0.07^{\mathrm{m}}$ & $0.41 \pm 0.03^{\text {ghijklm }}$ & $0.42 \pm 0.06^{\text {fghi }}$ & $0.24 \pm 0.05^{\mathrm{klm}}$ & $0.28 \pm 0.03^{\mathrm{ijklm}}$ & $0.28 \pm 0.03^{\mathrm{ijkkm}}$ & $0.25 \pm 0.01^{\mathrm{jklm}}$ & $0.35 \pm 0.01^{\text {hijklm }}$ & $0.01 \pm 0.00^{\mathrm{n}}$ \\
\hline \multicolumn{11}{|c|}{ Area $\left(\mathrm{cm}^{2}\right)$ Leaf $^{-1}$} \\
\hline Control & $20.00 \pm 0.58^{\mathrm{c}}$ & $13.34 \pm 0.69^{\mathrm{fgh}}$ & $12.25 \pm 0.89^{\text {ghij }}$ & $15.60 \pm 0.74 \mathrm{de}$ & $14.79 \pm 0.41^{\text {ef }}$ & $14.91 \pm 0.55^{\text {ef }}$ & $12.59 \pm 0.47 \mathrm{ghi}$ & $7.63 \pm 0.15^{\text {opqrs }}$ & $27.33 \pm 0.88^{\mathrm{a}}$ & $8.53 \pm 0.34^{\text {nopqr }}$ \\
\hline Mild & $17.00 \pm 0.58^{\mathrm{d}}$ & $11.82 \pm 0.32 \mathrm{hij}$ & $12.17 \pm 0.58$ ghij & $13.91 \pm 0.36^{\mathrm{efg}}$ & $11.38 \pm 0.35^{\mathrm{ijk}}$ & $10.94 \pm 0.88^{\mathrm{ijkl}}$ & $10.79 \pm 0.76^{\mathrm{ijklm}}$ & $6.56 \pm 0.53^{\text {rstu }}$ & $23.37 \pm 0.32^{\mathrm{b}}$ & $7.17 \pm 0.18^{\text {pqrst }}$ \\
\hline Moderate & $11.33 \pm 0.88^{\mathrm{ijk}}$ & $8.73 \pm 0.44^{\text {nopq }}$ & $9.77 \pm 0.79 \mathrm{klmn}$ & $9.35 \pm 0.34^{\mathrm{Imno}}$ & $8.51 \pm 0.71$ nopqr & $9.00 \pm 0.58^{\text {mnop }}$ & $9.78 \pm 0.53 \mathrm{klmn}$ & $6.17 \pm 0.15^{\text {stu }}$ & $20.00 \pm 0.58^{\mathrm{c}}$ & $5.98 \pm 0.39^{\text {stu }}$ \\
\hline Severe & $10.63 \pm 0.55^{\mathrm{jklm}}$ & $7.27 \pm 0.92^{\text {pqrst }}$ & $7.41 \pm 0.89^{\text {opqrst }}$ & $5.65 \pm 0.42^{\text {tuv }}$ & $7.00 \pm 0.47^{\text {qrst }}$ & $7.15 \pm 0.69^{p}$ & $7.92 \pm 0.56^{\text {nopqrs }}$ & $5.03 \pm 0.93$ uv & $16.76 \pm 0.39^{\mathrm{d}}$ & $4.10 \pm 0.32^{\mathrm{v}}$ \\
\hline
\end{tabular}

Means followed by a similar letter within a column for each parameter are not significantly different at the 0.05 level of probability by Duncan's Multiple-Range Test. 
Table 2. Effect of drought stress on leaf relative water content (RWC), proline (Pro) content and total chlorophyll (Total Chlo) content in faba bean genotypes.

\begin{tabular}{|c|c|c|c|c|c|c|c|c|c|c|}
\hline \multirow{2}{*}{ Treatments } & \multicolumn{10}{|c|}{ Genotypes } \\
\hline & Zafar 1 & Zafar 2 & Giza Blanka & Espan & Makamora & Shebam 1 & Giza 3 & $\mathrm{C} 4$ & $\mathrm{C5}$ & G853 \\
\hline \multicolumn{11}{|c|}{ RWC \% } \\
\hline Control & $75.19 \pm 0.36^{\mathrm{de}}$ & $78.15 \pm 0.28^{b}$ & $73.23 \pm 0.29^{f}$ & $71.05 \pm 0.69^{\mathrm{g}}$ & $73.03 \pm 0.33^{\mathrm{f}}$ & $76.51 \pm 0.76^{\mathrm{cd}}$ & $69.14 \pm 0.10^{\mathrm{h}}$ & $76.89 \pm 0.44^{\mathrm{bc}}$ & $80.84 \pm 0.33^{\text {a }}$ & $62.71 \pm 0.55^{\mathrm{n}}$ \\
\hline Mild & $64.89 \pm 0.50^{\mathrm{lm}}$ & $70.84 \pm 0.53 \mathrm{~g}$ & $66.14 \pm 0.89^{\mathrm{ijkl}}$ & $69.24 \pm 0.44^{\mathrm{h}}$ & $71.24 \pm 0.58^{g}$ & $64.14 \pm 0.66^{\mathrm{m}}$ & $65.20 \pm 0.50 \mathrm{klm}$ & $65.09 \pm 0.38 \mathrm{klm}$ & $74.03 \pm 0.13^{\text {ef }}$ & $53.89 \pm 0.05^{\mathrm{r}}$ \\
\hline Moderate & $66.92 \pm 0.33 \mathrm{ij}$ & $66.26 \pm 0.37^{\mathrm{ijkl}}$ & $60.96 \pm 0.70^{\circ}$ & $66.51 \pm 0.59^{\mathrm{ijk}}$ & $55.71 \pm 0.60^{9}$ & $57.18 \pm 0.69^{p}$ & $60.92 \pm 0.30^{\circ}$ & $60.20 \pm 0.55^{\circ}$ & $68.12 \pm 0.23 \mathrm{hi}$ & $50.13 \pm 0.18^{\text {tu }}$ \\
\hline Severe & $34.48 \pm 0.46^{z}$ & $44.39 \pm 0.30^{\mathrm{w}}$ & $49.19 \pm 0.11$ uv & $41.79 \pm 0.20^{x}$ & $45.26 \pm 0.45 \mathrm{w}$ & $48.15 \pm 0.11^{\mathrm{v}}$ & $50.87 \pm 0.26^{\mathrm{st}}$ & $38.23 \pm 0.94^{y}$ & $52.09 \pm 0.14^{\mathrm{s}}$ & $25.82 \pm 0.65^{z}$ \\
\hline \multicolumn{11}{|c|}{$\operatorname{Pro}\left(\mu \mathrm{g}^{-1} \cdot \mathrm{FW}\right)$} \\
\hline Control & $0.91 \pm 0.01^{\mathrm{opq}}$ & $1.30 \pm 0.02^{\mathrm{hijkl}}$ & $0.90 \pm 0.06^{\mathrm{pq}}$ & $1.05 \pm 0.02^{\text {Imnopq }}$ & $0.56 \pm 0.03^{\mathrm{r}}$ & $0.89 \pm 0.07^{\mathrm{pq}}$ & $0.91 \pm 0.03^{\text {opq }}$ & $1.21 \pm 0.03 \mathrm{i}^{\mathrm{jklmn}}$ & $1.34 \pm 0.01^{\text {hijk }}$ & $0.49 \pm 0.02^{\mathrm{r}}$ \\
\hline Mild & $1.11 \pm 0.01$ klmnopq & $1.85 \pm 0.02^{\mathrm{f}}$ & $1.17 \pm 0.03^{\mathrm{ij} k \mathrm{j} m n o p}$ & $1.21 \pm 0.09^{\mathrm{e}}$ & $0.99 \pm 0.01 \mathrm{mnopq}$ & $0.91 \pm 0.05^{\circ \mathrm{opq}}$ & $1.10 \pm 0.05^{\mathrm{klmnopq}}$ & $1.45 \pm 0.01 \mathrm{hi}$ & $2.42 \pm 0.02^{\mathrm{d}}$ & $0.51 \pm 0.03^{\mathrm{r}}$ \\
\hline Moderate & $1.78 \pm 0.03 \mathrm{fg}$ & $2.28 \pm 0.06^{\mathrm{de}}$ & $1.24 \pm 0.01^{\mathrm{ijklm}}$ & $2.11 \pm 0.05^{\mathrm{ij} k \mathrm{lmn}}$ & $1.19 \pm 0.02^{\mathrm{ijk} k m n o}$ & $1.19 \pm 0.01^{\mathrm{ijk} k m n o}$ & $1.21 \pm 0.07^{\mathrm{ij} k \mathrm{klmn}}$ & $1.55 \pm 0.01^{\text {gh }}$ & $2.82 \pm 0.01^{\mathrm{c}}$ & $0.84 \pm 0.01^{\mathrm{fg}}$ \\
\hline Severe & $2.29 \pm 0.11 \mathrm{de}$ & $3.11 \pm 0.42^{b}$ & $1.43 \pm 0.02^{\text {hijk }}$ & $2.53 \pm 0.03^{\mathrm{d}}$ & $1.82 \pm 0.08^{\mathrm{f}}$ & $1.52 \pm 0.17^{\mathrm{h}}$ & $1.16 \pm 0.01^{\mathrm{jklmnop}}$ & $2.82 \pm 0.03^{\mathrm{c}}$ & $3.65 \pm 0.06^{\mathrm{a}}$ & $0.94 \pm 0.01^{\text {nopq }}$ \\
\hline \multicolumn{11}{|c|}{ Total $C h l\left(\mathrm{mg}^{-1} \mathrm{~g}^{-1} \cdot \mathrm{FW}\right)$} \\
\hline Control & $40.47 \pm 0.53^{\text {cde }}$ & $40.07 \pm 0.74^{\mathrm{cde}}$ & $40.87 \pm 0.59^{\mathrm{cd}}$ & $41.50 \pm 0.42^{\mathrm{bcd}}$ & $38.77 \pm 0.67^{\mathrm{efg}}$ & $37.43 \pm 0.76^{\text {gh }}$ & $38.00 \pm 0.06^{\mathrm{f}}$ & $41.97 \pm 0.24 \mathrm{bcd}$ & $46.77 \pm 0.59^{\mathrm{a}}$ & $35.00 \pm 0.58^{j}$ \\
\hline Mild & $37.27 \pm 0.62$ ghi & $38.00 \pm 0.85^{\mathrm{fg}}$ & $35.40 \pm 0.23^{\mathrm{ij}}$ & $35.37 \pm 0.35 \mathrm{ij}$ & $38.00 \pm 0.58^{\mathrm{fg}}$ & $32.77 \pm 0.19^{\mathrm{kl}}$ & $37.67 \pm 0.88^{\mathrm{fg}}$ & $39.67 \pm 0.39^{\text {def }}$ & $42.80 \pm 0.23^{b}$ & $27.00 \pm 0.58^{p}$ \\
\hline Moderate & $35.60 \pm 0.60^{\mathrm{hij}}$ & $32.33 \pm 0.88^{1}$ & $34.43 \pm 0.70^{\mathrm{jk}}$ & $32.40 \pm 0.83^{1}$ & $32.83 \pm 0.44^{\mathrm{kl}}$ & $29.27 \pm 0.43^{\text {no }}$ & $32.57 \pm 0.78^{\mathrm{kl}}$ & $35.67 \pm 0.93^{\mathrm{hij}}$ & $37.80 \pm 0.46^{\mathrm{fg}}$ & $23.67 \pm 0.88^{q}$ \\
\hline Severe & $32.00 \pm 0.58^{1}$ & $26.83 \pm 0.44^{\mathrm{p}}$ & $27.80 \pm 0.20^{\mathrm{op}}$ & $29.90 \pm 0.90^{\mathrm{mn}}$ & $31.03 \pm 0.98^{\mathrm{lmn}}$ & $29.27 \pm 0.73^{\text {no }}$ & $32.23 \pm 0.62^{1}$ & $29.73 \pm 0.37 \mathrm{mn}$ & $31.43 \pm 0.431^{\mathrm{m}}$ & $18.67 \pm 0.88^{\mathrm{r}}$ \\
\hline
\end{tabular}

Means followed by a similar letter within a column for each parameter are not significantly different at the 0.05 level of probability by Duncan's Multiple-Range Test. 
Table 3. Effect of drought stress on electrolyte leakage and content of malondialdehyde (MDA) and hydrogen peroxide $\left(\mathrm{H}_{2} \mathrm{O}_{2}\right)$ in faba bean genotypes.

\begin{tabular}{|c|c|c|c|c|c|c|c|c|c|c|}
\hline \multirow{2}{*}{ Treatments } & \multicolumn{10}{|c|}{ Genotypes } \\
\hline & Zafar 1 & Zafar 2 & Giza Blanka & Espan & Makamora & Shebam 1 & Giza 3 & $\mathrm{C4}$ & $\mathbf{C 5}$ & G853 \\
\hline \multicolumn{11}{|c|}{ Electrolyte Leakage (\%) } \\
\hline Control & $32.67 \pm 1.45^{\mathrm{n}}$ & $37.00 \pm 1.15^{\mathrm{n}}$ & $35.33 \pm 1.45^{\mathrm{n}}$ & $36.00 \pm 1.53^{n}$ & $37.67 \pm 1.45^{\mathrm{n}}$ & $43.33 \pm 1.76^{\mathrm{m}}$ & $37.33 \pm 1.20^{\mathrm{n}}$ & $37.67 \pm 1.45^{\mathrm{n}}$ & $35.00 \pm 0.58^{\mathrm{n}}$ & $47.00 \pm 1.15^{\mathrm{klm}}$ \\
\hline Mild & $45.00 \pm 2.65^{\mathrm{m}}$ & $46.67 \pm 1.20^{\mathrm{klm}}$ & $46.33 \pm 0.88^{\mathrm{lm}}$ & $48.00 \pm 1.53^{\mathrm{klm}}$ & $55.33 \pm 1.20^{\mathrm{ij}}$ & $55.33 \pm 1.76^{\mathrm{ij}}$ & $51.33 \pm 1.86^{\mathrm{jkl}}$ & $45.33 \pm 1.45^{\mathrm{m}}$ & $45.00 \pm 2.65^{\mathrm{m}}$ & $57.67 \pm 1.45^{\text {hi }}$ \\
\hline Moderate & $55.67 \pm 2.03^{\mathrm{ij}}$ & $55.33 \pm 2.03^{\mathrm{ij}}$ & $53.67 \pm 2.33^{\mathrm{ij}}$ & $64.00 \pm 2.65^{\mathrm{efg}}$ & $66.00 \pm 1.73^{\text {defg }}$ & $66.00 \pm 2.08^{\text {defg }}$ & $57.67 \pm 0.88^{\mathrm{hi}}$ & $61.67 \pm 1.20^{\mathrm{gh}}$ & $51.67 \pm 2.03^{\mathrm{jk}}$ & $66.67 \pm 1.67$ defg \\
\hline Severe & $66.00 \pm 1.53^{\text {defg }}$ & $67.00 \pm 1.15^{\text {def }}$ & $69.00 \pm 0.58^{\text {cde }}$ & $70.33 \pm 0.88^{\text {cd }}$ & $76.33 \pm 2.19^{\mathrm{ab}}$ & $72.33 \pm 1.20^{\mathrm{bc}}$ & $67.67 \pm 1.33^{\text {cde }}$ & $76.00 \pm 1.53^{\text {ab }}$ & $62.33 \pm 1.45^{\text {fgh }}$ & $77.67 \pm 1.45^{\text {a }}$ \\
\hline \multicolumn{11}{|c|}{ MDA Content $\left(\mathrm{nmol}^{-} \mathrm{g}^{-1} \cdot \mathbf{F W}\right)$} \\
\hline Control & $27.33 \pm 1.20^{\mathrm{n}}$ & $27.00 \pm 1.15^{\mathrm{n}}$ & $24.67 \pm 2.03^{n}$ & $26.00 \pm 1.53^{\mathrm{n}}$ & $27.67 \pm 1.45^{\mathrm{n}}$ & $33.33 \pm 1.76^{\mathrm{m}}$ & $25.00 \pm 0.58^{\mathrm{n}}$ & $27.67 \pm 1.45^{\mathrm{n}}$ & $22.33 \pm 1.45^{\mathrm{n}}$ & $37.00 \pm 1.15^{\mathrm{lm}}$ \\
\hline Mild & $41.33 \pm 1.86^{\mathrm{kl}}$ & $36.67 \pm 1.20^{\mathrm{lm}}$ & $36.33 \pm 0.88^{\mathrm{lm}}$ & $38.00 \pm 1.53^{\mathrm{lm}}$ & $45.33 \pm 1.20^{\mathrm{jk}}$ & $45.33 \pm 1.76^{\mathrm{jk}}$ & $35.00 \pm 2.65^{\mathrm{m}}$ & $35.33 \pm 1.45^{\mathrm{m}}$ & $35.00 \pm 2.65^{\mathrm{m}}$ & $47.67 \pm 1.45^{\mathrm{ij}}$ \\
\hline Moderate & $45.67 \pm 2.03^{\mathrm{jk}}$ & $45.33 \pm 2.03^{\mathrm{jk}}$ & $51.67 \pm 1.20^{\mathrm{hi}}$ & $54.00 \pm 2.65^{\text {fgh }}$ & $56.00 \pm 1.73^{\text {efgh }}$ & $56.00 \pm 2.08^{\text {efgh }}$ & $47.67 \pm 0.88^{\mathrm{ij}}$ & $43.67 \pm 2.33^{\mathrm{jk}}$ & $45.00 \pm 1.73^{\mathrm{jk}}$ & $57.67 \pm 1.45^{\text {defg }}$ \\
\hline Severe & $55.33 \pm 2.03^{\text {efgh }}$ & $60.33 \pm 0.88^{\text {cde }}$ & $58.33 \pm 1.20^{\mathrm{def}}$ & $62.33 \pm 1.20^{\mathrm{bcd}}$ & $64.33 \pm 2.60$ abc & $55.33 \pm 1.86^{\text {efgh }}$ & $57.67 \pm 1.33^{\text {defg }}$ & $67.00 \pm 1.53^{a b}$ & $52.33 \pm 1.45^{\text {ghi }}$ & $67.67 \pm 1.45^{\text {a }}$ \\
\hline \multicolumn{11}{|c|}{$\mathrm{H}_{2} \mathrm{O}_{2}$ Content $\left(\mathrm{m} \mu \cdot \mathrm{mol} \mathrm{g}^{-1} \cdot\right.$ leaf $\left.\cdot \mathrm{FW}\right)$} \\
\hline Control & $15.00 \pm 0.58^{q}$ & $18.67 \pm 0.88^{\circ}$ & $16.00 \pm 0.58^{\mathrm{pq}}$ & $18.00 \pm 1.15^{\mathrm{op}}$ & $18.00 \pm 0.58^{\mathrm{op}}$ & $18.67 \pm 0.88^{\circ}$ & $20.00 \pm 0.58^{\text {no }}$ & $19.00 \pm 0.58^{\circ}$ & $15.00 \pm 0.58^{q}$ & $18.67 \pm 0.33^{\circ}$ \\
\hline Mild & $23.33 \pm 0.88^{\mathrm{lm}}$ & $24.67 \pm 0.67^{\mathrm{kl}}$ & $22.00 \pm 1.15^{\mathrm{mn}}$ & $24.00 \pm 0.58^{\mathrm{lm}}$ & $20.33 \pm 0.88^{\text {no }}$ & $26.00 \pm 0.58^{\mathrm{jkl}}$ & $27.00 \pm 1.15^{\mathrm{jk}}$ & $25.00 \pm 0.58^{\mathrm{kl}}$ & $19.00 \pm 0.58^{\circ}$ & $25.33 \pm 0.88^{\mathrm{kl}}$ \\
\hline Moderate & $29.67 \pm 0.88^{\mathrm{hi}}$ & $28.33 \pm 0.88^{\mathrm{ij}}$ & $29.67 \pm 0.88^{\mathrm{hi}}$ & $30.00 \pm 1.15^{\mathrm{hi}}$ & $26.00 \pm 1.15^{\mathrm{jkl}}$ & $31.00 \pm 0.58^{\mathrm{gh}}$ & $33.33 \pm 0.88^{\text {efg }}$ & $27.00 \pm 0.58^{\mathrm{jk}}$ & $23.33 \pm 0.88^{\mathrm{lm}}$ & $32.00 \pm 1.15^{\text {fgh }}$ \\
\hline Severe & $34.67 \pm 0.88^{\text {cde }}$ & $33.67 \pm 0.88^{\text {def }}$ & $35.00 \pm 0.58^{\text {cde }}$ & $37.67 \pm 0.88^{b}$ & $31.33 \pm 0.88^{\text {fgh }}$ & $36.00 \pm 0.58^{\mathrm{bcd}}$ & $37.00 \pm 0.58^{\mathrm{bc}}$ & $31.67 \pm 0.88^{\text {fgh }}$ & $28.33 \pm 0.88^{\mathrm{ij}}$ & $43.67 \pm 0.88^{\mathrm{a}}$ \\
\hline
\end{tabular}

Means followed by a similar letter within a column for each parameter are not significantly different at the 0.05 level of probability by Duncan's Multiple-Range Test. 
Table 4. Effect of drought stress on the activity of catalase (CAT), peroxidase (POD) and superoxide dismutase (SOD) in faba bean genotypes.

\begin{tabular}{|c|c|c|c|c|c|c|c|c|c|c|}
\hline \multirow{2}{*}{ Treatments } & \multicolumn{10}{|c|}{ Genotypes } \\
\hline & Zafar 1 & Zafar 2 & Giza Blanka & Espan & Makamora & Shebam 1 & Giza 3 & $\mathrm{C4}$ & C5 & G853 \\
\hline \multicolumn{11}{|c|}{ CAT Activity (units $\cdot \mathrm{mg}^{-1} \cdot$ protein $\cdot \mathrm{min}^{-1}$ ) } \\
\hline Mild & $148.7 \pm 1.76^{\mathrm{op}}$ & $178.0 \pm 1.73^{\text {hi }}$ & $156.0 \pm 2.08^{\mathrm{n}}$ & $167.7 \pm 3.76^{\mathrm{klm}}$ & $172.3 \pm 1.20^{\mathrm{jk}}$ & $171.0 \pm 2.08^{\mathrm{jk}}$ & $197.0 \pm 2.31^{\mathrm{f}}$ & $181.3 \pm 1.86^{\mathrm{hi}}$ & $206.0 \pm 3.06^{\mathrm{e}}$ & $154.7 \pm 2.60^{\text {no }}$ \\
\hline Moderate & $164.7 \pm 1.76^{\mathrm{lm}}$ & $195.0 \pm 1.73^{\mathrm{f}}$ & $163.0 \pm 2.08^{\mathrm{m}}$ & $178.0 \pm 1.53^{\text {hi }}$ & $182.3 \pm 1.45^{\mathrm{h}}$ & $184.0 \pm 1.53^{\mathrm{h}}$ & $194.0 \pm 5.13^{\mathrm{f}}$ & $209.0 \pm 3.21^{\mathrm{e}}$ & $222.7 \pm 1.45^{\mathrm{cd}}$ & $163.3 \pm 2.03^{\mathrm{m}}$ \\
\hline Severe & $247.7 \pm 1.45^{\mathrm{a}}$ & $204.3 \pm 2.33^{\mathrm{e}}$ & $185.3 \pm 2.03^{\mathrm{gh}}$ & $216.3 \pm 4.10^{\mathrm{d}}$ & $194.0 \pm 2.31^{\mathrm{f}}$ & $223.7 \pm 2.73^{\mathrm{c}}$ & $220.3 \pm 1.20^{\mathrm{cd}}$ & $237.3 \pm 2.33^{\mathrm{b}}$ & $242.7 \pm 1.76$ ab & $175.0 \pm 1.73^{\mathrm{ij}}$ \\
\hline Control & $20.50 \pm 0.23^{\mathrm{kl}}$ & $20.33 \pm 0.32^{\mathrm{lm}}$ & $13.70 \pm 0.12^{q}$ & $16.27 \pm 0.27^{\circ}$ & $19.83 \pm 0.15^{\mathrm{lm}}$ & $11.27 \pm 0.27^{\mathrm{r}}$ & $16.43 \pm 0.24^{\circ}$ & $9.87 \pm 0.47^{\mathrm{s}}$ & $21.33 \pm 0.23^{\mathrm{jk}}$ & $13.63 \pm 0.18^{\mathrm{q}}$ \\
\hline Mild & $23.10 \pm 0.31^{\text {hi }}$ & $23.30 \pm 0.31^{\text {hi }}$ & $16.30 \pm 0.23^{\circ}$ & $19.50 \pm 0.17^{\mathrm{m}}$ & $23.50 \pm 0.21^{\mathrm{gh}}$ & $13.90 \pm 0.23^{\mathrm{q}}$ & $20.40 \pm 0.32^{\mathrm{lm}}$ & $11.93 \pm 0.47^{\mathrm{r}}$ & $24.67 \pm 0.12^{\text {ef }}$ & $15.70 \pm 0.26^{\mathrm{op}}$ \\
\hline Moderate & $25.37 \pm 0.18^{\mathrm{e}}$ & $25.53 \pm 0.23^{\mathrm{e}}$ & $19.77 \pm 0.47^{\mathrm{lm}}$ & $21.60 \pm 0.15^{\mathrm{j}}$ & $26.57 \pm 0.18^{\mathrm{d}}$ & $18.50 \pm 0.23^{\mathrm{n}}$ & $24.30 \pm 0.26^{\mathrm{fg}}$ & $15.30 \pm 0.26^{\mathrm{p}}$ & $27.23 \pm 0.24^{\text {bcd }}$ & $20.40 \pm 0.32^{\mathrm{lm}}$ \\
\hline Severe & $27.50 \pm 0.23^{\mathrm{bc}}$ & $26.67 \pm 0.30^{\mathrm{cd}}$ & $24.47 \pm 0.85^{\mathrm{f}}$ & $24.33 \pm 0.32^{\mathrm{fg}}$ & $28.10 \pm 0.12^{b}$ & $20.53 \pm 0.23^{\mathrm{kl}}$ & $26.53 \pm 0.23^{\mathrm{d}}$ & $18.30 \pm 0.29^{\mathrm{n}}$ & $29.50 \pm 0.21^{\mathrm{a}}$ & $22.47 \pm 0.20^{\mathrm{i}}$ \\
\hline \multicolumn{11}{|c|}{ SOD Activity (units $\cdot \mathrm{mg}^{-1} \cdot \operatorname{protein} \cdot \mathrm{min}^{-1}$ ) } \\
\hline Control & $9.33 \pm 0.33^{\text {rst }}$ & $10.00 \pm 0.58$ qrs & $11.00 \pm 0.58^{\mathrm{pqr}}$ & $6.00 \pm 0.58^{\mathrm{v}}$ & $8.00 \pm 0.58^{\text {tu }}$ & $7.00 \pm 0.58$ uv & $8.67 \pm 0.33^{\text {stu }}$ & $7.33 \pm 0.33$ uv & $11.33 \pm 0.33^{\mathrm{pq}}$ & $14.00 \pm 0.58^{\mathrm{mn}}$ \\
\hline Moderate & $20.00 \pm 0.58^{\text {efg }}$ & $19.00 \pm 0.58^{\text {ghi }}$ & $20.00 \pm 0.58^{\text {efg }}$ & $18.00 \pm 0.58^{\text {hij }}$ & $17.67 \pm 0.88^{\mathrm{ijk}}$ & $14.00 \pm 0.58^{\mathrm{mn}}$ & $16.00 \pm 1.15^{\mathrm{kl}}$ & $16.33 \pm 0.33^{\mathrm{jkl}}$ & $21.33 \pm 0.88^{\text {def }}$ & $18.67 \pm 0.33^{\mathrm{ghi}}$ \\
\hline Severe & $25.00 \pm 0.58^{\mathrm{b}}$ & $24.00 \pm 0.58^{\mathrm{bc}}$ & $25.00 \pm 0.58^{b}$ & $22.67 \pm 0.88^{\mathrm{cd}}$ & $21.67 \pm 0.88^{\text {de }}$ & $24.33 \pm 0.33^{b c}$ & $21.00 \pm 0.58^{\text {def }}$ & $19.67 \pm 0.33^{\mathrm{fgh}}$ & $28.00 \pm 0.58^{\mathrm{a}}$ & $20.33 \pm 0.33^{\text {efg }}$ \\
\hline
\end{tabular}

Means followed by a similar letter within a column for each parameter are not significantly different at the 0.05 level of probability by Duncan's Multiple-Range Test. 


\section{Experimental Section}

\subsection{Plant and Treatment}

Seeds of 10 improved genotypes of Vicia faba L. were obtained from different geographical origins. Seeds of genotypes Zafar 1, Zafar 2 and Shebam from the General organization for Agriculture Research, Yemen, genotypes Makamora and Espan from the local market of Riyadh and genotypes Giza Blanka, Giza 3, C4, C5 and G853 from Agriculture Research Center, Egypt. The experiment was conducted in a growth chamber (temperature $25 \pm 3{ }^{\circ} \mathrm{C}$, relative humidity $50 \%-60 \%$, light $250 \mu \mathrm{mol}$ of photons $\mathrm{m}^{-2} \cdot \mathrm{s}^{-1}$ on a $16 / 8$-h light/dark cycle). Seeds were grown in pots containing a mixture of sand and peat (1:1). Drought stress was initiated when seedlings attained 2-3 true leaves. Drought stress treatments were imposed by withholding water. The details of the drought stress treatments were as follows: (i) Control (UN): Normal irrigation (irrigated alternate day); (ii) Mild stress (D1): Irrigation after 3 days of control plants irrigation; (iii) Moderate stress (D2): Irrigation after 6 days of control plants irrigation; and (iv) Severe stress (D3): Irrigation after 9 days of control plants irrigation. The experimental pots were arranged in a simple randomized design with five replicates per treatment. Before sowing, seeds of all genotypes were surface sterilized with $1 \%$ sodium hypochlorite for $10 \mathrm{~min}$, then were vigorously rinsed with double distilled water (DDW) and sown in sand+peat-filled pots supplied with Raukura's nutrient solution [34]. The salts used to make up the nutrient solution are as follows: Macronutrient stock solution $\mathrm{A}\left(\mathrm{g} \cdot \mathrm{L}^{-1}\right) \mathrm{Mg}\left(\mathrm{NO}_{3}\right)_{2} \cdot 6 \mathrm{H}_{2} \mathrm{O}, 4.94 ; \mathrm{Ca}\left(\mathrm{NO}_{3}\right)_{2} \cdot 4 \mathrm{H}_{2} \mathrm{O}, 16.78 ; \mathrm{NH}_{4} \mathrm{NO}_{3}, 8.48 ; \mathrm{KNO}_{3}, 2.28$. Macronutrient stock solution $\mathrm{B}\left(\mathrm{g} \cdot \mathrm{L}^{-1}\right) \mathrm{KH}_{2} \mathrm{PO}_{4}, 2.67 ; \mathrm{K}_{2} \mathrm{HPO}_{4}, 1.64 ; \mathrm{K}_{2} \mathrm{SO}_{4}, 6.62 ; \mathrm{Na}_{2} \mathrm{SO}_{4}, 0.60 ; \mathrm{NaCl}$, 0.33. Micronutrient supplement $\left(\mathrm{mg} \cdot \mathrm{L}^{-1}\right) \mathrm{H}_{3} \mathrm{BO}_{3}, 128.80 ; \mathrm{CuCl}_{2} \cdot 2 \mathrm{H}_{2} \mathrm{O}, 4.84 ; \mathrm{MnCl}_{2} \cdot 4 \mathrm{H}_{2} \mathrm{O}, 81.10$; $\left(\mathrm{NH}_{4}\right) 6 \mathrm{Mo}_{7} \mathrm{O}_{24} \cdot 4 \mathrm{H}_{2} \mathrm{O}, 0.83 ; \mathrm{ZnCl}_{2}, 23.45$; ferric citrate pentahydrate, 809.84 . The dilute solution which was applied to the plants was prepared by mixing $200 \mathrm{~mL}$ of each of the macronutrient stock solution with $100 \mathrm{~mL}$ of the micronutrient supplement and was diluted to $4.5 \mathrm{~L}$ with DDW.

Sampling was done after 50 days of sowing. The growth performance of faba bean plants was assessed in terms of plant height (PH) plant ${ }^{-1}$, fresh weight (FW) and dry weight (DW) plant ${ }^{-1}$ and area (A) leaf ${ }^{-1}$.

The leaf area was measured directly using Leaf Area Meter (Model LI-3050A, LI-COR Inc, Lincon, NE, USA). The area of three leaves (upper, middle, and lower) of each plant of the sample (consisting of five plants) was determined.

\subsection{Determination of Physio-Biochemical Characteristics}

\subsubsection{Leaf Relative Water Content}

Leaf RWC (\%) was determined using the methods of Gulen and Eris [35]. Leaf discs of $1.5 \mathrm{~cm}$ diameter were taken from the fully expanded and uniform leaves of each of the three plants (replicates) per treatment. First, the FW was recorded, and then samples were placed in a petri dish having distilled water for $4 \mathrm{~h}$. Turgid weight (TW) was then recorded, and the leaf samples were placed in an incubator at $70{ }^{\circ} \mathrm{C}$ for $24 \mathrm{~h}$, to determine the dry weight. Leaf RWC \% was calculated by:

$$
\mathrm{RWC}(\%)=[(\mathrm{FW}-\mathrm{DW}) /(\mathrm{TW}-\mathrm{DW})] \times 100
$$




\subsubsection{Total Chlorophyll Concentration}

The youngest fully expanded leaves were subjected to extraction using $80 \%$ acetone, and the absorbance was measured using UV-vis Spectrophotometer (SPEKOL 1500; Analytik Jena AG, Jena, Germany) at 663 and $645 \mathrm{~nm}$. The total chlorophyll content was determined by using Arnon's formula [36].

\subsubsection{Proline Concentration}

The proline concentration was determined spectrophotometrically using the ninhydrin method of Bates et al. [37]. First, fresh leaf samples were homogenized in 3\% sulfosalicylic acid, followed by the addition of $2 \mathrm{~mL}$ each of ninhydrin and glacial acetic acid, after which the samples were heated to $100{ }^{\circ} \mathrm{C}$. The mixture was then extracted with toluene, and the free toluene was quantified at $520 \mathrm{~nm}$.

\subsubsection{MDA Concentration}

The MDA content was determined according to the method of Heath and Packer [38]. Leaf samples were weighed, and homogenates containing $10 \%$ trichloroacetic acid and $0.65 \%$ 2-thiobarbituric acid were heated at $95{ }^{\circ} \mathrm{C}$ for $60 \mathrm{~min}$, then cooled to room temperature, and centrifuged at $10,000 \times g$ for $10 \mathrm{~min}$. The absorbance of the supernatant was read at 532 and $600 \mathrm{~nm}$ against a reagent blank.

\subsubsection{Electrolyte Leakage}

Electrolyte leakage was used to assess membrane permeability in accordance with Lutts et al. [39]. Samples were washed 3 times with double-distilled water to remove surface contamination, and leaf discs were cut from young leaves and placed in sealed vials containing $10 \mathrm{~mL}$ of DDW, followed by incubation on a rotary shaker for $24 \mathrm{~h}$, after which the electrical conductivity of the solution (EC1) was determined. Then, the samples were autoclaved at $120^{\circ} \mathrm{C}$ for $20 \mathrm{~min}$, and the electrical conductivity was measured again (EC2) after the solution was cooled to room temperature. The electrolyte leakage was defined as EC1/EC2 $\times 100$ and expressed as percentage.

\subsubsection{Hydrogen Peroxide $\left(\mathrm{H}_{2} \mathrm{O}_{2}\right)$}

It was measured as described by Velikova et al. [40]. Fresh leaf samples $(0.5 \mathrm{~g})$ were homogenized in $5 \mathrm{~mL}$ of $0.1 \%(w / v)$ TCA. The homogenate was centrifuged at $12,000 \mathrm{rpm}$ for $15 \mathrm{~min}$ and the supernatant was added to $10 \mathrm{mM}$ potassium phosphate buffer $(\mathrm{pH}$ 7.0) and $1 \mathrm{M}$ potassium iodide. The absorbance of the supernatant was recorded at $390 \mathrm{~nm}$. The content of $\mathrm{H}_{2} \mathrm{O}_{2}$ was calculated by comparison with a standard calibration curve plotted using known concentrations of $\mathrm{H}_{2} \mathrm{O}_{2}$.

\subsection{Determination of Antioxidant Enzymes' Activity}

To determine the activities of antioxidant enzymes, a crude enzyme extract was prepared by homogenizing $500 \mathrm{mg}$ of leaf tissue in extraction buffer $(0.5 \%$ Triton X-100 and $1 \%$ polyvinylpyrrolidone in $100 \mathrm{mM}$ potassium phosphate buffer, $\mathrm{pH}$ 7.0) using a chilled mortar and pestle. The homogenate was then centrifuged at $15,000 \times \mathrm{g}$ for $20 \mathrm{~min}$ at $4{ }^{\circ} \mathrm{C}$, and the supernatant was used for the enzymatic assays described below. All enzyme activities were expressed as milligram of protein per minute. 
We applied the method of Chance and Maehly [41] to determine POD (EC 1.11.1.7) activity using $5 \mathrm{~mL}$ of enzyme reaction solution containing phosphate buffer ( $\mathrm{pH} \mathrm{6.8),} 50 \mathrm{M}$ pyrogallol, $50 \mathrm{mM} \mathrm{H}_{2} \mathrm{O}_{2}$, and $1 \mathrm{~mL}$ of the enzyme extract diluted 20 times. The assay mixture was incubated for $5 \mathrm{~min}$ at $25{ }^{\circ} \mathrm{C}$, and the reaction was terminated by the addition of $0.5 \mathrm{~mL}$ of $5 \%(v / v) \mathrm{H}_{2} \mathrm{SO}_{4}$. Purpurogallin production was measured spectrophotometrically at $420 \mathrm{~nm}$. One unit of POD activity was considered the amount of purpurogallin formed per milligram of protein per minute.

The method of Aebi [42] was used to measure CAT (EC 1.11.1.6) activity. The decomposition of $\mathrm{H}_{2} \mathrm{O}_{2}$ was measured as the decrease in absorbance at $240 \mathrm{~nm}$. In this assay, $50 \mathrm{mM}$ phosphate buffer ( $\mathrm{pH} 7.8$ ) and $10 \mathrm{mM} \mathrm{H}_{2} \mathrm{O}_{2}$ were used in the reaction solution.

Activity of SOD (EC 1.15.1.1) was determined based on the inhibition of nitro blue tetrazolium (NBT) photoreduction according to the method of Giannopolitis and Ries [43]. The reaction solution (3 mL) contained $50 \mathrm{mM}$ NBT, $1.3 \mathrm{mM}$ riboflavin, $13 \mathrm{mM}$ methionine, $75 \mu \mathrm{M}$ ethylenediamine tetraacetic acid (EDTA), $50 \mathrm{mM}$ phosphate buffer (pH 7.8), and 20 to $50 \mathrm{~mL}$ of enzyme extract. The reaction solution was irradiated under fluorescent light at $75 \mu \mathrm{M} \cdot \mathrm{m}^{-2} \cdot \mathrm{s}^{-1}$ for $15 \mathrm{~min}$. The absorbance at $560 \mathrm{~nm}$ was read against a blank (non-irradiated reaction solution). One unit of SOD activity was defined as the amount of enzyme that inhibited $50 \%$ of NBT photoreduction.

\subsection{Statistical Analysis}

The data were expressed as the mean \pm standard error and were analyzed statistically using IBM SPSS Ver.22 statistical software (IBM Corporation and Others, Armonk, NY, USA). The means were compared statistically using Duncan's multiple-range test at the level of $p<0.05$.

\section{Conclusions}

All morphological, physiological and biochemical characteristics of 10 genotypes of faba bean reduced under drought stress. We observed all genotypes of faba bean behaved differently under water stress. In the present study, we found some of the genotypes were tolerant ("C5" and "Zafar 1"), mild tolerant ("Giza 3", "Zafar 2", "Giza Blanka", "Espan”, "Shebam 1" and "Makamora”) and sensitive ("G853" and "C4") to water stress. However, genotypes "C5" and "Zafar 1" performed better by improving RWC and accumulation of Pro. Also, tolerant genotypes had a better ability to reduce oxidative damage by increasing activity of CAT, POD and SOD. For further study, theses genotypes can be used to uncover molecular mechanism(s) involved in building the tolerance of faba bean plants to drought stress.

\section{Acknowledgments}

This project was supported by NSTIP Strategic Technologies Program, number (11-BIO1922-02) in the Kingdom of Saudi Arabia. 


\section{Author Contributions}

Manzer H. Siddiqui, Mohamed H. Al-Whaibi and Anil Grover conceived and designed experiment; Manzer H. Siddiqui, Mutahhar Y. Al-Khaishany and Mohammed A. Al-Qutami performed experiment; Mutahhar Y. Al-Khaishany and Hayssam M. Ali analyzed the data; Mona S. Al-Wahibi and Najat A. Bukhari collected seeds and contributed materials; Manzer H. Siddiqui and Mohamed H. Al-Whaibi wrote manuscript.

\section{Conflicts of Interest}

The authors declare no conflict of interest.

\section{References}

1. Blum, A. Drought resistance-Is it really a complex trait? Funct. Plant Biol. 2011, 38, 753-757.

2. Zlatev, Z.; Lidon, F.C. An overview on drought induced changes in plant growth, water relations and photosynthesis. Emir. J. Food Agric. 2012, 24, 57-72.

3. De Carvalho, M.H.C. Drought stress and reactive oxygen species: Production, scavenging and signaling. Plant Signal. Behav. 2008, 3, 156-165.

4. Cornic, G. Drought stress inhibits photosynthesis by decreasing stomatal aperture: Not by affecting ATP synthesis. Trend Plant Sci. 2000, 5, 187-188.

5. Parry, M.A.J.; Androlojc, P.J.; Khan, S.; Lea, P.J.; Keys, A.J. Rubisco activity: Effects of drought stress. Ann. Bot. 2002, 89, 833-839.

6. Bota, J.; Medrano, H.; Flexas, J. Is photosynthesis limited by decreased Rubisco acivity and RuBP content under progressive water stress? New Phytol. 2004, 162, 671-681.

7. Jaleel, C.A.; Sankar, B.; Murali, P.V.; Gomathinayagam, M.; Lakshmanan, G.M.A.; Panneerselvam, R. Water deficit stress effects on reactive oxygen metabolism in Catharanthus roseus; Impacts on ajmalicine accumulation. Colloids Surf. B 2008, 62, 105-111.

8. Farooq, M.; Basra, S.M.A.; Wahid, A.; Cheema, Z.A.; Cheema, M.A.; Khaliq, A. Physiological role of exogenously applied glycinebetaine in improving drought tolerance of fine grain aromatic rice (Oryza. sativa L.). J. Agron. Crop Sci. 2008, 194, 325-333.

9. Shinozaki, K.; Yamaguchi-Shinozaki, K. Gene networks involved in drought stress response and tolerance. J. Exp. Bot. 2007, 58, 221-227.

10. Siddiqui, M.H.; Khan, M.N.; Mohammad, F.; Khan, M.M.A. Role of nitrogen and gibberellin (GA3) in the regulation of enzyme activities and in osmoprotectant accumulation in Brassica juncea L. under salt stress. J. Agron. Crop Sci. 2008, 194, 214-224.

11. Razmjoo, K.; Heydarizadeh, P.; Sabzalian, M.R. Effect of salinity and drought stresses on growth parameters and essential oil content of Matricaria. chamomile. Int. J. Agric. Biol. 2008, 10, 451-454.

12. Barnabás, B.; Jäger, K.; Fehér, A. The effect of drought and heat stress on reproductive processes in cereals. Plant Cell Environ. 2008, 31, 11-38.

13. FAOSTAT, Food and Agriculture Organization, 2009. Available online: http://faostat.fao.org/site/ 567/default.aspx\#ancor (accessed on 17 April 2015). 
14. Blair, M.W.; Soler, A.; Cortès A.J. Diversification and population structure in common beans (Phaseolus vulgaris L.). PLoS ONE 2012, 7, 10-11.

15. Cortès, A.J.; Monserrate, F.A.; Ramírez-Villegas, J.; Madriñán, S.; Blair M.W. Drought tolerance in wild plant populations: The case of common beans (Phaseolus. vulgaris L.). PLoS ONE 2013, 8 , $1-10$.

16. Cortés, A.J.; This, D.; Chavarro, C.; Madriñán, S.; Blair M.W. Nucleotide diversity patterns at the drought-related DREB2 encoding genes in wild and cultivated common bean (Phaseolus vulgaris L.). Theor. Appl. Genet. 2012, 125, 1069-1085.

17. Loss, S.P.; Siddique, K.H.M. Adaptation of faba bean (Vicia faba L.) to dryland Mediterranean-type environments I. Seed yield and yield components. Field Crop. Res. 1997, 53, 17-28.

18. Farooq, M.; Hussain, M.; Wahid, A.; Siddique, K.H.M. Drought stress in plants: An overview. In Plant Responses to Drought Stress from Morphological to Molecular Features; Aroca, R., Ed.; Springer-Verlag: Berlin, Germany, 2012; pp. 1-36.

19. Yordanov, I.; Velikova, V.; Tsonev, T. Plant responses to drought and stress tolerance. Bulg. J. Plant Physiol. 2003, Special Issue 2003, 187-206.

20. Ouzounidou, G.; Ilias, I.F.; Giannakoula, A.; Theocharidou I. Effect of water stress and $\mathrm{NaCl}$ triggered changes on yield, physiology, biochemistry of broad bean (Vicia faba L.) plants and on quality of harvested pods. Biologia 2014, 69, 1010-1017.

21. Ali, H.M.; Siddiqui, M.H.; Al-Whaibi, M.H.; Basalah, M.O.; Sakran, A.M.; El-Zaidy, M. Effect of proline and abscisic acid on the growth and physiological performance of faba bean under water stress. Pak. J. Bot. 2013, 45, 933-940.

22. Asrar, A.W.A.; Elhindi, K.M. Alleviation of drought stress of marigold (Tagetes. erecta) plants by using arbuscular mycorrhizal fungi. Saudi J. Biol. Sci. 2011, 18, 93-98.

23. Ritchie, S.W.; Nguyan, H.T.; Holaday, A.S. Leaf Water content and gas exchange parameters of two wheat genotypes differing in drought resistance. Crop. Sci. 1990, 30, 105-111.

24. Zhang, L.; Gao, M.; Hu, J.; Zhang, X.; Wang, K.; Ashraf, M. Modulation role of abscisic acid (ABA) on growth, water relations and glycinebetaine metabolism in two maize (Zea. mays L.) cultivars under drought stress. Int. J. Mol. Sci. 2012, 13, 3189-3202

25. Khanna-Chopra, R.; Selote, D.S. Acclimation to drought stress generates oxidative stress tolerance in drought-resistant than-susceptible wheat cultivar under field conditions. Environ. Exp. Bot. 2007, 60, 276-283.

26. Blum A.; Ebercon, A. Genotypic responses in sorghum to drought stress. III. Free proline accumulation and drought resistance. Crop Sci. 1976, 16, 428-431.

27. Mafakheri, A.; Siosemardeh, A.; Bahramnejad, B.; Struik, P.C.; Sohrabi, Y. Effect of drought stress on yield, proline and chlorophyll contents in three chickpea cultivars. Aust. J. Crop Sci. 2010, 4, 580-585.

28. Reddy, M.P.; Vora, A.B. Changes in pigment composition, Hill reaction activity and saccharides metabolism in Bajra (Pennisetum typhoides $\mathrm{S} \& \mathrm{H}$ ) leaves under $\mathrm{NaCl}$ salinity. Photosynthetica 1986, 20, 50-55.

29. Terzi, R.; Kadioglu, A. Drought stress tolerance and the antioxidant enzyme system in Ctenanthe setosa. Acta Biol. Crac. Ser. Bot. 2006, 48, 89-96. 
30. Quan, R.; Shang, M.; Zhang, H.; Zhao, Y.; Zhang, J. Engineering of enhanced glycine betaine synthesis improves drought tolerance in maize. Plant Biotechnol. J. 2004, 2, 477-486.

31. Jiang, Y.; Huang, B. Drought and heat stress injury to two cool-season turfgrasses in relation to antioxidant metabolism and lipid peroxidation. Crop Sci. 2001, 41, 436-442.

32. Foyer, C.H.; Fletcher, J.M. Plant antioxidants: Colour me healthy. Biologist 2001, 48, 115-120.

33. Siddiqui, M.H.; Al-Whaibi, M.H.; Sakran, A.M.; Basalah, M.O.; Ali H.M. Effect of calcium and potassium on antioxidant system of Vicia faba L. under cadmium stress. Int. J. Mol. Sci. 2012, 13, 6604-6619.

34. Smith, G.S.; Johnston, C.M.; Cornforth, I.S. Comparison of nutrient solutions for growth of plants in sand culture. New Phytol. 1983, 94, 537-548.

35. Gulen, H.; Eris, A. Some physiological changes in strawberry (Fragaria $\times$ Ananassa. cv. "Camarosa") plants under heat stress. J. Hort. Sci. Biotechnol. 2003, 78, 894-898.

36. Arnon, D.I. Copper enzymes in isolated chloroplast. Polyphenol oxidase in Beta vulgaris. Plant Physiol. 1949, 24, 1-15.

37. Bates, L.S.; Waldren, R.P.; Teare, I.D. Rapid determination of free proline for water-stress studies. Plant Soil 1973, 9, 205-207.

38. Heath, R.L.; Packer, L. Photoperoxidation in isolated chloroplasts. I. Kinetics and stoichiometry of fatty acid peroxidation. Arch. Biochem. Biophys. 1968, 125, 189-198.

39. Lutts, S.; Kinet, J.M.; Bouharmont, J. Changes in plant response to $\mathrm{NaCl}$ during development of rice (Oryza sativa L.) varieties differing in salinity resistance. J. Exp. Bot. 1995, 46, 1843-1852.

40. Velikova, V.; Yordanov, I.; Edreva, A. Oxidative stress and some antioxidant systems in acid rain-treated bean plants: Protective role of exogenous polyamines. Plant Sci. 2000, 151, 59-66.

41. Chance, B.; Maehly, A.C. Assay of catalase and peroxidases. Methods Enzymol. 1955, 2, 764-775.

42. Aebi, H. Catalase in vitro. Methods Enzymol. 1984, 105, 121-126.

43. Giannopolitis, C.N.; Ries, S.K. Superoxide dismutases: I. Occurrence in higher plants. Plant Physiol. 1977, 59, 309-314.

(C) 2015 by the authors; licensee MDPI, Basel, Switzerland. This article is an open access article distributed under the terms and conditions of the Creative Commons Attribution license (http://creativecommons.org/licenses/by/4.0/). 